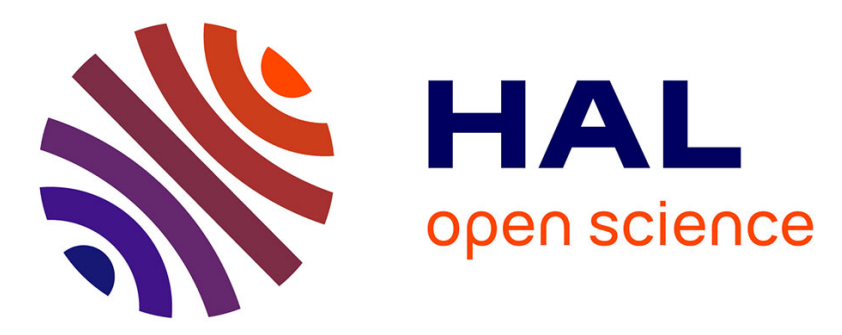

\title{
MSW Signal-to-Noise Enhancers for Noise Reduction in DBS Reception
}

\author{
T. Nomoto, T. Kuki
}

\section{To cite this version:}

T. Nomoto, T. Kuki. MSW Signal-to-Noise Enhancers for Noise Reduction in DBS Reception. Journal de Physique IV Proceedings, 1997, 07 (C1), pp.C1-387-C1-390. 10.1051/jp4:19971157 jpa-00254802

\section{HAL Id: jpa-00254802 https://hal.science/jpa-00254802}

Submitted on 1 Jan 1997

HAL is a multi-disciplinary open access archive for the deposit and dissemination of scientific research documents, whether they are published or not. The documents may come from teaching and research institutions in France or abroad, or from public or private research centers.
L'archive ouverte pluridisciplinaire HAL, est destinée au dépôt et à la diffusion de documents scientifiques de niveau recherche, publiés ou non, émanant des établissements d'enseignement et de recherche français ou étrangers, des laboratoires publics ou privés. 


\title{
MSW Signal-to-Noise Enhancers for Noise Reduction in DBS Reception
}

\author{
T. Nomoto and T. Kuki \\ NHK Science and Technical Research Laboratories, 1-10-11 Kinuta, Setagaya-ku, Tokyo 157, Japan
}

\begin{abstract}
The RF performance of MSW signal- to- noise enhancers is characterized by operational power level and frequency, frequency-selectivity, and transient response in MSW saturation. The frequency selectivity and transient response characteristics are especially important for noise reduction in frequency-modulated signals such as DBS reception signals. However, it is difficult to make both the characteristics good at the same time. In this paper three types of signal-to-noise enhancers with difference structures are discussed from the viewpoint of DBS application. We show that using an MSBVW/SW hybrid mode is an effective approach to lowering the operational frequency.
\end{abstract}

\section{INTRODUCTION}

MSW (magnetostatic wave) devices such as delay lines, tesonators and filters have been investigated in the past 20-30 years. The nonlinearity of MSWs, especially the MSSW (magnetostatic surface wave) mode has contributed unique devices such as signal-tonoise enhancers and frequency-selective limiters [1], [2]. However, lack of themal stability and the necessity for a bias magnetic field have hindered enhancers in applications to signal processing. As a result, until now a devices for practical use has not been reported.

DBS (direct broadcast satellite) systems using a small antenna (less than $40 \mathrm{~cm}$ in diameter) have recently become very popular in Japan (more than 10 million receivers). Carrier- to- noise ratio $(\mathrm{C} / \mathrm{N})$ teduction of the received signal caused by rain attenuation frequentiy generates significant impairment of a TV picture in rainy countries like Japan. In the DBS system frequency-modulated (FM) signals are handled differently from noise reduction in frequency memory loops, where only fixed frequency signals are handled. If the signal-to-noise enhancers act as a narrow bandpass filter automatically tracking an instantaneous frequency of the FM signal, significant noise reduction is expected. We have therefore paid attention to the application of signal-to-noise enhancers to compensation for C/N reduction in DBS reception, even if they have some defects. To achieve the noise reduction, the enhancers must have a short transient response in time in MSW saturation as well as good frequency selectivity. However, making both characteristics good at the same time is because they have a contrary relationship to input power [3]. Another important condition is whether the enhancer can be easily applied to the current DBS receiving system, where frequencies of $403 \mathrm{MHz}$ with a $27-\mathrm{MHz}$ bandwidth and 1 to $1.3 \mathrm{GHz}$ are used as the IF bands (Fig. 1). In addition, low-power operation is needed to lower the power range of the enhancer driving circuit.

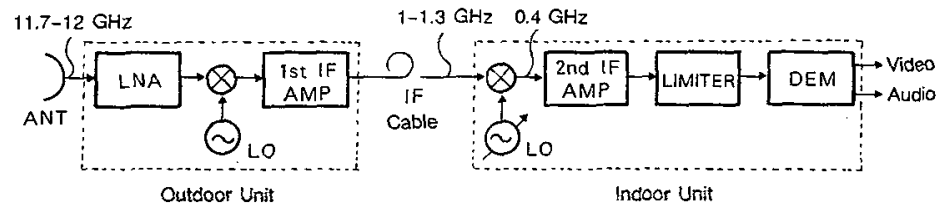

Fig. 1 Block diagram of the current DBS receiver. 
The signal- to- noise enhancers can be formed with three different types of structures. We call them the transmission enhancer [1], the cancellation enhancer [3] and the reflection enhancer [5]. The transmission type enhancer has the most simple structure of the three, followed by the reflection enhancer and then the cancellation enhancer. However, because they display a different RF performance, the most suitable enhancer should be selected according to the application without reference to the structure.

In this paper the above three types of enhancers are compared from the viewpoint of an application to the current DBS receiving system. It also discusses using an MSBVW/SW (magnetostatic backward volume wave and surface wave) hybrid mode to make the operational frequency lower.

\section{DESIGN}

Figures 2(a), 2(b) and 2(c) show the geometries of the different types of MSW signal-to-noise enhancers using MSSW modes. In operation of the transmission enhancer [Fig. 2(a)], a microwave input signal gradually launches MSWs into the YIG film along the microstrip line from the input toward the output. The microwave signal is then attenuated at the output for a small signal input. However, for a large signal input above the threshold level, conversion from the microwave to MSWs saturates resulting in no more attenuation of the microwave signal. In this enhancer the conversion may not be limited near the output, even if the conversion is saturated near the input, and then a steep enhancement characteristic may not be anticipated. The sum of the enhancement is determined by the length of the YIG film contact with the microstrip line.

The cancellation enhancer [Fig. 2(b)] comprises two MSW filters, two $90^{\circ}$ hybrids and two attenuators. A microwave input signal is divided into two signals by the hybrid 1, one of which directly goes to the MSW filter 2, while the other goes to MSW filter 1 through the Att 1 . Both the signals through filters 1 and 2 are recombined by the hybrid 2 with a $180^{\circ}$ phase difference resulting in combination with equal amplitude and opposite phase for a small signal input. The signals therefore cancel each other out and no signal appears at the output. However, for a large signal input above the threshold of filter 2 , the loss of path 2 is much larger than that of path 1, resulting in combination without equal amplitude. Thus, most of the signal through path 1 appears at the output. This enhancer achieves infinite enhancement and excellent thermal stability theoretically, if both the filters have the exactly same characteristics.

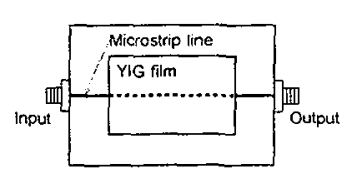

(a)

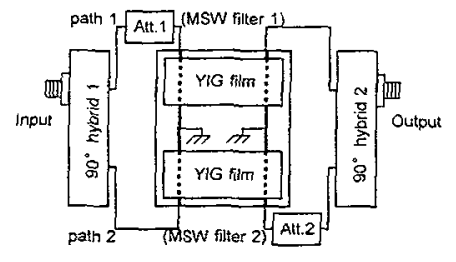

(b)

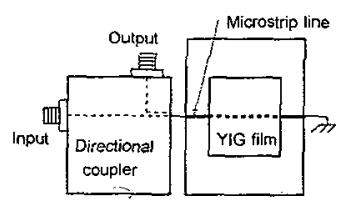

(c)

Fig. 2 Geometries of the signal-to-noise enhancers. (a) Transmission type. (b) Cancellation type. (c) Reflection type.

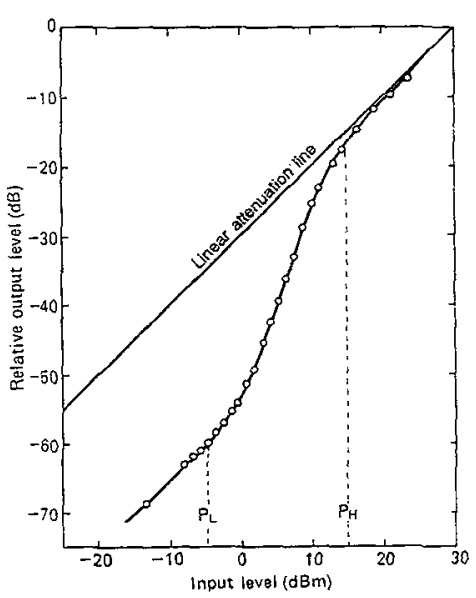

(a)

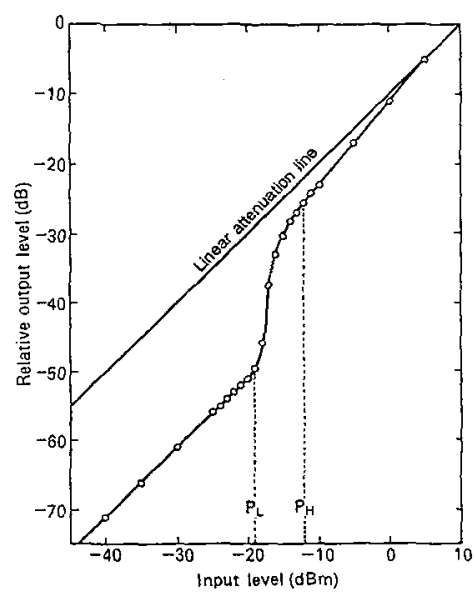

(b)

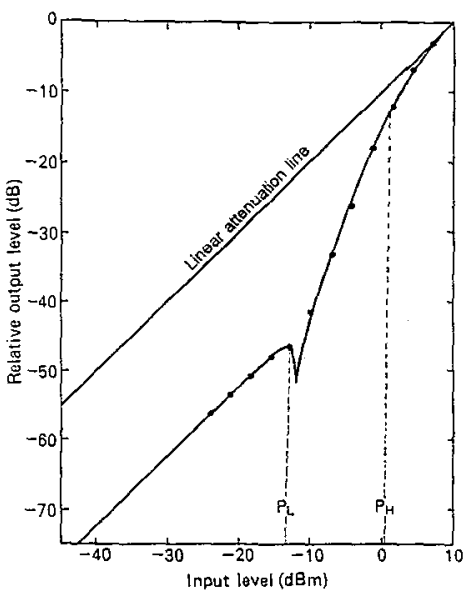

(c)

Fig. 3 Typical input-to-output relationships of the enhancers. (a) Transmission type. (b) Cancellation type. (c) Reflection type. 
The reflection enhancer [Fig. 2(c)] comprises a microstrip line, one terminal of which is short-circuited, and a directional coupler (or a circulator). Its operation is similar to that of the transmission enhancer, and only a reflected signal is extracted as an output signal through the directional coupler. The sum of the enhancement depends heavily on the matching of the microwave and MSWs, but does not depend too much on the length of the YIG film. This is a significant difference from the transmission enhancer.

Thus all these circuits frequency-selectively exhibit a high loss for small signals and a low loss for large signals; then they are called a signal- to- noise enhancer. The typical RF performance and effects on the DBS application are explained in the following sections.

\section{RF PERFORMANCE}

The three types of signal- to- noise enhancers exhibit a different RF performance. Figures 3(a), 3(b) and 3(c) show the typical inputoutput relationships of the enhancers, which have about an enhancement of $20 \mathrm{~dB}$. The smaller the level difference between $P_{H}$ and $P_{L}$ is, the lower the $C / N$ signal can be handled because we usually set a desired signal above $P_{H}$ and set noise below $P_{L}\left(P_{H}\right.$ and $P_{l}$. are defined in Ref. 3). If the absolute level of $\mathrm{P}_{H}$ is low, the power driving the enhancer also becomes small. In this viewpoint, the cancellation enhancer is best and the transmission enhancer may not be so good. We should be careful about the absolute level at the output because of the large insertion loss by the MSW filter and the attenuator.

Figures 4(a) and 4(b) are spectra showing frequency selectivity at the enhancer output, and Figures $5(\mathrm{a})$ and $5(\mathrm{~b})$ are oscilloscope traces showing transient response of the enhancer when the input microwave is pulse-modulated [3]. The frequency selectivity ( $B$ a) conduces to reduction of noise neighboring a desired signal. The transient response in time ( $\mathrm{T}_{\mathrm{s}}$ ), which is caused by conversion from MSWs to half- frequency spin waves excited in the YIG film, determines the ability to track instantaneous frequency shift of the signal. During the time $T_{S}$, a desired signal as well as noise is reduced resulting in no improvement of the signal $\mathrm{C} / \mathrm{N}$. With an increase of input power, $B_{a}$ increases but $T_{s}$ decreases. This shows that the product $U_{p}$ of $B a$ and $T_{s}$ is approximately constant and that $U_{p}$ is in proportion to the MSW group- velocity $V_{g}$ [4]. The $U_{p}$ should be as small as possible to achieve a good transient response. Controlling $V_{g}$ while the $M S W s$ propagate from the input to the output inside the filter is relatively easyt in the cancellation enhancer (changing the distribution of a bias magnetic field, for example). From this viewpoint the cancellation enhancer may also be best.

The IF frequencies handled for the current DBS receivers, on the other hand, are the 403- $\mathrm{MHz}$ and 1- to 1.3-(3Hz bands. The enhancer should directly operate in these bands without frequency conversion. However, MSSW modes are generally not used in operational frequencies below $2 \mathrm{GHz}$ (bias magnetic field is below $100 \mathrm{Oe}$ ) because of random domain formation caused by incomplete magnetic saturation in a pure YIG film with saturation magnetization of $1780 \mathrm{G}$. Reduction of the saturation magnetization is a good approach to lowering the operational frequency. Another is using a combination of MSBVW and MSSW modes; that is, an MSBVW/SW hybrid mode, where the magnetic bias field is applied between the directions for the pure MSBVW and MSSW modes. Figure 6 shows the reflection characteristic of the MSW filter using the hybrid mode. We found that with a

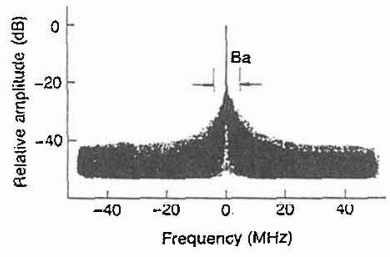

(a)

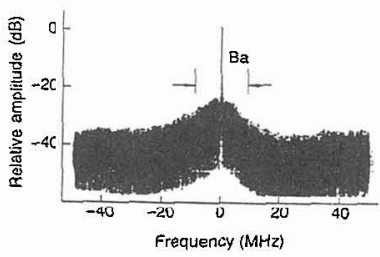

(b)

Fig. 4 Spectra showing the frequency selectivity of the enhancer. (a) Input power of $-6 \mathrm{dBm}$.

(b) Input power of $+4 \mathrm{dBm}$.

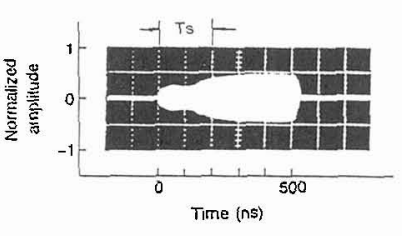

(a)

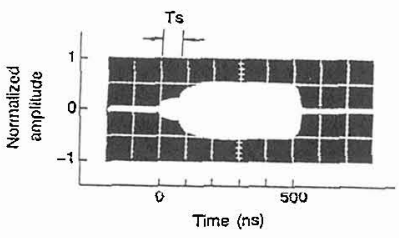

(b)

Fig. 5 Oscilloscope traces showing the transient response of the enhancer. (a) Input power of $-6 \mathrm{dBm}$.

(b) Input power of $+4 \mathrm{dBm}$. 


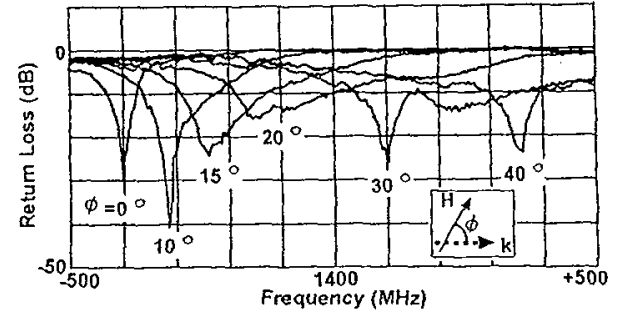

Fig. 6 Reflection of the MSW filter using the MSBVW/SW hybrid mode.

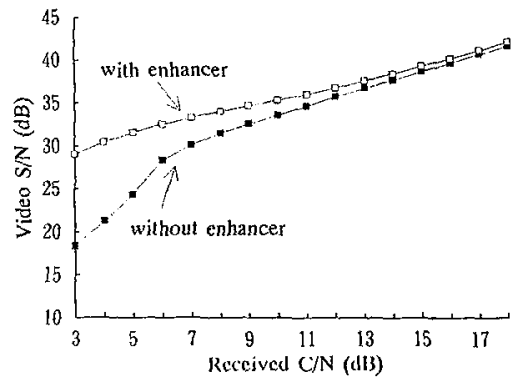

Fig. 7 Relationship between the received $\mathrm{C} / \mathrm{N}$ and the video S/N.

decrease in the angle $\phi\left(\phi=0^{\circ}\right.$ for the pure MSBVW mode), the matching (small reflection) frequency shifts lower. A small reflection means that efficient conversion from the microwave to the MSWs is made. At the same time we showed that $\mathrm{v}_{\mathrm{g}}$ becomes slow theoretically using the hybrid modes. In this way the hybrid mode is a very attractive approach for low frequency operation and good tracking ability. We should thoroughly investigate the hybrid mode.

\section{EFFECTS ON NOISE REDUCTION IN DBS RECEPTION}

We have developed signal-to-noise enhancers operating in the $403-\mathrm{MHz}$ and $1-$ to $1.3-\mathrm{GHz}$ bands using MSSW modes propagating into doped YIG films with saturation magnetization of $360 \mathrm{G}[5]$ and $1320 \mathrm{O}$ [3], respectively. Figure 7 shows the relationship between a received signal $\mathrm{C} / \mathrm{N}$ and a video $\mathrm{S} / \mathrm{N}$ measured by the 1- to 1.3-GHz cancellation enhancer. Below the FM threshold of $9 \mathrm{~dB}$, the $\mathrm{S} / \mathrm{N}$ is enhanced by 2 to $10 \mathrm{~dB}$; thus this enhancer performed what is called $\mathrm{FM}$ threshold extension. Even above the $\mathrm{C} / \mathrm{N}$ of $9 \mathrm{~dB}$, a slight enhancement of 1 to $2 \mathrm{~dB}$ is obtained. Little improvement is achieved in the densely colored parts of a TV picture, such as the red part in the color bar of the NTSC video signal, where the color-subcarrier ( $3.58 \mathrm{MHz}$ ) amplitude is large. In these parts, the carrier frequency of an FM signal is shifted rapidly and largely by the color-subcarrier. This is probably because the transient response in the MSW saturation is not so quick, and so the reduction of $\mathrm{U}_{\mathrm{p}}$ is needed for more improvement. In practice, however, densely colored parts rarely appear in normal TV pictures and therefore our developed enhancer is very effective in noise reduction in DBS reception. If the $\mathrm{C} / \mathrm{N}$ is enhanced by $3 \mathrm{~dB}$, the size of the receiving antenna is reduced to $1 / \sqrt{ } 2$. The 1- to $1.3 \mathrm{GHz}$ enhancer is stable despite temperature changes and the driving power is only $-5 \mathrm{~dB}$; thus it is suitable for practical use and will be commercially available in the very near future. The size of the practical enhancer is $18 \times 18 \times 11 \mathrm{~cm}^{3}$.

Also, a similar performance can be obtained in the $403-\mathrm{MHz}$ enhancer. We cannot but adopt the reflection enhancer, because the transmission loss inside the MSW filter is too large (over $50 \mathrm{~dB}$ ) and the driving power needs to be as small as possible. The details of this enhancer are reported in Ref. 5. The $403-\mathrm{MHz}$ enhancer operates with a driving power below $0 \mathrm{~dB}$, but it is too sensitive to temperature change. The thermal stability of the enhancer must be improved first for practical use. To this end, we are now considering optimizing the saturation magnetization of the YIG film by using the MSBVW/SW hybrid mode.

\section{CONCLUSION}

We have compared three types of signal- to- noise enhancer with different structures. The results showed that the cancellation enhancer was best for noise reduction in DBS application. Cancellation and reflection enhancers were respectively developed for the 1- to-1.3- $\mathrm{GHz}$ and 403- $\mathrm{MHz}$ bands for noise reduction. It was shown that the video $\mathrm{S} / \mathrm{N}$ was significantly improved and the 1- to 1.3-GHz enhancer was suitable for practical use. We also explained that using the MSBVW/SW hybrid mode was effective in reducing the operational frequency of the enhancers.

\section{References}

[1] J. D. Adam, IEEE Trans. Magnetics, Vol, MAG-16, No. 5, pp. 1168-1170 (1980)

[2] S. N. Stitzer and H. Goldie, 1983 IEEE MTT-S Dig. , 326-328.

[3] T. Nomoto and Y. Matsushita, IEEE Trans. Microwave Theory and Tech, Vol. 41, No. 8, pp.1316-1322 (1993).

[4] T. Kuki and T. Nomoto, 1994 Asia-Pacific Microwave Conference (APMC'94) Proc. , pp. 795-798.

[5] T. Kuki and T. Nomoto, 1995 IEEE MTT-S Dig. , pp.111-114. 